\title{
ADULTO SOBREVIVENTE DE CÂNCER INFANTIL: UMA REVISÃO BIBLIOGRÁFICA
}

\author{
Shirley Santos Teles \\ Elizabeth Ranier Martins do Valle
}

\begin{abstract}
RESUMO. Este artigo caracteriza-se como estudo bibliográfico e objetivou investigar, na literatura científica do período de janeiro de 2000 a abril 2007, artigos que abordam a temática de adultos sobreviventes a câncer infantil, a fim de traçar um panorama do que está sendo pesquisado e publicado na área, verificando a existência de tendências e possíveis lacunas. A coleta de dados foi realizada através da busca eletrônica de artigos indexados nas bases de dados Lilacs, Dedalus/Sibi, Scielo, Medline, PsycInfo a partir das palavras: "adult and survivor and child and cancer". As 36 publicações trabalhadas delinearamse em quatro enfoques: aspectos psicossociais, abrangendo estudos sobre qualidade de vida, estresse pós-traumático, sequelas psicológicas e hospitalizações psiquiátricas; saúde física/comprometimento físico; segunda neoplasia; e fertilidade e reprodução. A maioria dos artigos objetiva conhecer o funcionamento psicossocial do sobrevivente de câncer infantil. Percebe-se uma tendência apontando que os sobreviventes apresentam funcionamento psicossocial sem nenhum tipo de comprometimento.
\end{abstract}

Palavras-chave: Câncer infantil; adulto; sobrevivente.

\section{ADULT SURVIVORS OF CHILD CANCER: A LITERATURE REVIEW}

\begin{abstract}
This article presents a bibliographic review and aimed to search, in the scientific literature published between January 2000 and April 2007, articles on adult survivors of child cancer, in order to sketch a panorama of what is being published in the area, verifying the existence of tendencies and possible gaps. Data were collected through the electronic search of articles indexed in the databases Lilacs, Dedalus/Sibi, Scielo, Medline, PsycInfo, using the words: "adult and survivor and child and cancer". The 36 publications included were outlined in four focus areas: psychosocial aspects, covering research on quality of life, post-traumatic stress, psychological sequelae, psychiatric internments; physical health/physical problem; second tumor; fertility and reproduction. Most articles aim to get to know the psychosocial functioning of child cancer survivors. A certain trend is perceived, indicating that the survivors' psychosocial functioning is not affected.
\end{abstract}

Key words: Child cancer; adult; survivor.

\section{ADULTO SOBREVIVIENTE DE CÁNCER INFANTIL: UNA REVISIÓN BIBLIOGRÁFICA}

RESUMEN. Este artículo es caracterizado como estudio bibliográfico y objetivó investigar la literatura científica en el período de enero de 2000 a abril de 2007, artículos que tratan de la temática de adultos sobrevivientes de cáncer infantil, a fin de trazar un panorama de lo que está siendo investigado y publicado en el área, verificando la existencia de tendencias y posibles lagunas. La recolecta de datos fue realizada a través de la búsqueda electrónica de artículos indexados en las bases de datos Lilacs, Dedalus/Sibi, Scielo, Medline, PsycInfo, a partir de las palabras: "adult and survivor and child and cancer". Las 36 publicaciones trabajadas se delinearon en cinco enfoques: aspectos psicosociales, abarcando estudios sobre calidad de vida, estrés posttraumático, secuelas psicológicas, hospitalizaciones psiquiátricas; salud física/ comprometimiento físico; segunda neoplasía; fertilidad y reproducción. La mayoría de los artículos visa conocer el funcionamiento psicosocial del sobreviviente de cáncer infantil. Se percibe una cierta tendencia apuntando que los sobrevivientes presentan funcionamiento psicosocial sin comprometimiento.

Palabras-clave: Cáncer infantil; adulto; sobreviviente.

* Psicóloga, Mestre em Psicologia pela Faculdade de Filosofia, Ciências e Letras de Ribeirão Preto - Universidade de São Paulo e Doutoranda em Psicologia pela Faculdade de Filosofia, Ciências e Letras de Ribeirão Preto-USP.

\# Psicóloga, Professora Livre-docente do Departamento de Enfermagem Psiquiátrica e Ciências Humanas da Escola de Enfermagem de Ribeirão Preto - Universidade de São Paulo. 
O câncer é uma doença rara na idade infantil e na primeira fasze da adolescência, afetando cerca de 10 em 100.000 crianças a cada ano. Considerando-se crianças com até 15 anos, isso significa que uma em cada setecentas desenvolverá câncer durante esse período (Voûte, 1999).

A evolução das modalidades terapêuticas combinadas com cirurgia, radioterapia e quimioterapia, aliada à centralização e melhor suporte de cuidado, tem contribuído para um aumento linear das taxas de cura do câncer infantil. No Brasil, as crianças e jovens com leucemia linfática aguda (LLA), que corresponde a um terço dos tipos de câncer infantil, curam-se em $70 \%$ a $80 \%$ dos casos (Instituto Nacional do Câncer, 2007).

Apesar dos diferentes tipos de câncer e das diferentes formas de tratamento, estima-se que a taxa de cura global esteja em torno de $85 \%$. É esperado que, em 2010, um em cada 250 adultos seja sobrevivente de câncer na infância (Steuber \& Nesbit, 1997 citados em Rodrigues \& Camargo, 2003).

Atualmente já se sabe que a sobrevivência por cinco anos tem sido somente o início, e não o ponto final do sucesso do tratamento. Diante desta realidade, começaram a ser realizadas pesquisas com o intuito de conhecer os efeitos tardios, assim como o funcionamento psicossocial do adulto sobrevivente a câncer infantil. Não obstante, por ser essa área relativamente nova tanto no campo teórico quanto no âmbito assistencial, os dados encontrados ainda não são sistemáticos, sendo necessários estudos que reúnam esses achados para que se possa ter uma noção geral do que vem sendo encontrado (Hewitt, Weiner \& Simone, 2003; Schwartz, 2003).

Além disso, a sistematização desses achados é necessária para se poder pensar em serviços de acompanhamento a sobreviventes de câncer infantil que sejam efetivos e acolham as demandas dessa população.

\section{OBJETIVO}

O presente estudo teve como objetivo realizar um levantamento de artigos oublicados na literatura científica no período de janeiro de 2000 a abril de 2007 que abordam a temática de adultos sobreviventes de câncer infantil, a fim de traçar um panorama do que está sendo pesquisado e publicado na área e verificar as tendências e possíveis lacunas.

\section{METODOLOGIA}

O método utilizado para a coleta de dados foi o levantamento bibliográfico através da busca eletrônica de artigos indexados nas bases de dados Lilacs (Literatura Latino-Americana de Ciências de Saúde), Dedalus/Sibi (que contém o acervo de obras da Universidade de São Paulo), Scielo (Scientific Eletronic Library Online), Medline (United States National Library of Medicine), PsycInfo (base de dados da American Psychological Association) a partir das palavras: "adult and survivor and child and cancer", nas bases internacionais; "adulto e sobrevivente e câncer e infantil" nas bases nacionais.

Foram incluídos os artigos: a) publicados em periódicos indexados; b) escritos em língua inglesa, espanhola ou portuguesa; c) publicados entre janeiro de 2000 e abril de 2007; d) empíricos; e) que objetivassem estudar adultos sobreviventes de câncer infantil.

Diante da diversidade dos trabalhos encontrados, fez-se necessário estabelecer alguns critérios de exclusão: trabalhos que abordassem a questão familiar; validação de instrumento; capítulos de livros, teses, dissertações, publicações tipo carta, comentário ou editorial; artigos sobre adolescentes e crianças sobreviventes de câncer infantil; estudos que abordassem questões estritamente médicas e temáticas como fumo, obesidade e alimentação; referências sem resumo; trabalhos que abordassem doenças pediátricas de uma maneira geral; artigos que objetivassem avaliar serviços para acompanhamento de sobreviventes.

\section{RESULTADOS}

Foram encontrados 946 artigos na base de dados Medline. Com base nos critérios de inclusão e de exclusão das referências, foram selecionados 169 artigos. Destes, 69 eram artigos empíricos. Estes artigos abrangiam de 1994 a 2007. Com o recorte de anos de janeiro de 2000 a abril de 2007, foram selecionados 32 trabalhos, sendo recuperados $26 . \mathrm{Na}$ base de dados PsycInfo foram encontrados 41 artigos entre os anos de 1986 e 2007, dos quais 22 eram empíricos. Entre os anos de 2000 e 2007 foram encontrados 11 artigos, sendo recuperados 10. Assim, foram recuperados 36 artigos provenientes do Medline e do PsycInfo.

Nas bases de dados nacionais nenhum artigo foi encontrado.

Panorama geral do levantamento a partir dos resumos: breve delineamento histórico

Os primeiros resumos encontrados que objetivavam estudar adultos sobreviventes de câncer infantil datam do final da década de 80. Estes 
trabalhos, em sua maioria, são teóricos e discutem a questão da cura a longo prazo. Um estudo empírico iniciou a discussão sobre a intervenção psicológica para sobreviventes de câncer infantil (Cella et al., 1987). A questão da barreira para o emprego foi discutida teoricamente por Mellette e Franco (1987). Um artigo empírico foi desenvolvido com o intuito de investigar a decisão de casar e ter filhos com sobreviventes comparando-os com o grupo-controle de irmãos (Teeter, Holmes, Holmes \& Baker, 1987).

Os trabalhos que objetivavam estudar adultos sobreviventes de câncer infantil começaram efetivamente em meados da década de 90 (Weigers et al., 1998; Garre et al., 1994; Mulhern et al., 1995).

Estes dados refletem a trajetória do câncer infantil ao longo dos anos. De 1950 a 1960, sobreviventes de câncer eram raros, então o foco era como cuidar da criança que está morrendo. De 1970 a 1980 ainda havia a ameaça pela doença, mas a cura já era possível em muitos casos. Em torno de 1990, os estudos eram centrados no enfrentamento, na competência, bem como na sobrevivência de câncer a longo prazo. Em torno de 2000, começou a investigação dos efeitos tardios, com o intuito de identificar os fatores de risco (Eiser, 1998; Meadows, 2003).

\section{Análise dos artigos empíricos selecionados}

\section{Análise quantitativa}

No que concerne à metodologia adotada nos trabalhos, dos 36 artigos empíricos, apenas 1 (um) era qualitativo,1 (um) quantiqualitativo e os demais 34 estudos, quantitativos.

Com relação ao ano de publicação, quatro estudos foram realizados até abril de 2007, sete em 2006, cinco em 2005, cinco em 2004, oito em 2003, três em 2002, dois em 2001 e dois em 2000.

Dos 36 artigos selecionados, vinte e um foram realizados nos Estados Unidos, sete na Holanda, dois no Reino Unido e dois na Dinamarca, e o Canadá, a Espanha, a Alemanha, a Áustria e a Eslovênia publicaram 1(um) trabalho cada um.

No que tange ao periódico de publicação, a parcela maior -8 trabalhos - foi publicada na revista Psycho-oncology; e quanto às instituições de pesquisa, há uma predominância do Departamento de Pediatria da Universidade de Minnesota, nos Estados Unidos, que coordena o The Childhood Cancer Survivor Study (CCSS), que consiste em um banco de dados multi-institucional de sobreviventes. Os critérios de inclusão foram: o diagnóstico e o tratamento inicial terem sido realizados em uma das 25 instituições colaboradoras da CCSS, diagnosticados entre $1^{\circ}$ de janeiro 1970 e 31 de dezembro de 1986; ter até 21 anos no período do diagnóstico; e ter sobrevivido 5 anos desde o diagnóstico. Onze trabalhos pprovieram desta base de dados, representando $29 \%$ dos trabalhos empíricos localizados (Zebrack et al., 2002; Green et al., 2002; Nagarajan et al., 2003; Hudson et al., 2003; Zebrack et al., 2004; Park et al., 2005; Signorello et al., 2006; Oeffinger et al., 2006; Henderson et al., 2007).

Três estudos foram realizados na clínica de acompanhamento do Hospital Emma Kinderziekenhuis/Academic Medical Center em Amsterdã, na Holanda. Os critérios de inclusão foram ser sobrevivente com idade a partir de 16 anos e ter tido câncer até os 19 anos de idade (Langeveld, Grootenhuis, Voûte, Haan \& Van den Boss, 2004, Langeveld et al., 2003, Langeveld, Grootenhuis, Voûte \& Van den Boss, 2003).

Também no The Emma Children's Hospital/ Academic Medical Center, na Holanda, foi desenvolvido o VOLG- study, um estudo holandês sobre as consequências psicossociais a longo prazo do câncer na infância. Os critérios de inclusão utilizados foram: ter idade entre 18 e 30 anos no tempo do estudo; ter concluído o tratamento pelo menos 5 anos antes; ter sido diagnosticado até 18 anos. Este estudo gerou dois artigos (Stam, Grootenhuis \& Last, 2005, Stam, Grootenhuis, Caron \& Last, 2006).

No que concerne ao tipo de delineamento, todos os trabalhos desenvolvidos foram transversais.

Com relação aos sujeitos-participantes dos estudos, foi possível observar uma grande variedade no tocante aos critérios de inclusão: a idade no momento do estudo, idade no diagnóstico, quantidade de anos para ser considerado sobrevivente e início da contagem destes anos (no diagnóstico ou no término do tratamento). Com relação ao grupo-controle foram utilizados grupos de irmãos e grupos de população normal.

\section{Análise qualitativa}

Após a leitura dos artigos empíricos selecionados na íntegra, foi possível realizar uma análise qualitativa, dividindo os estudos em cinco grandes grupos:

- Aspectos psicossociais, que abrange estudos sobre qualidade de vida, estresse pós-traumático, sequelas psicológicas e hospitalizações psiquiátricas; B) Saúde física/ Comprometimento físico; C) Segunda neoplasia; D) Fertilidade $e$ reprodução.

\section{Aspectos psicossociais}

Cinco estudos investigaram a qualidade de vida do adulto sobrevivente de câncer infantil. Destes, três 
utilizaram o conceito de Health - Related Quality of Life (HRQoL), constructo psicológico multidimensional que inclui, pelo menos, quatro domínios: funcionamento físico, cognitivo, social e emocional. O HRQoL dos sobreviventes parece ser mais influenciado pelo bem-estar subjetivo do paciente do que pelos dados objetivos médicos (Pemberger et al., 2005). O HRQoL dos sobreviventes foi pior, se comparado ao grupo sem história de câncer, porém as diferenças foram bem pequenas (Stam et al., 2006). Quando comparado o HRQoL do adulto sobrevivente a longo termo (até 20 anos de idade) e a longo termo tardio (mais de 20 anos de idade), os sobreviventes a longo termo tardio têm maior porcentagem de efeitos tardios severos e percebem a qualidade de vida pior que os sobreviventes diagnosticados mais recentemente (Blaauwbroek et al., 2007).

A qualidade de vida dos adultos sobreviventes de câncer infantil foi relatada como positiva nos domínios físico, psicológico, social e espiritual, assim como a autoestima (Zebrack \& Chesler, 2002, Langeveld et al., 2004).

Entre os jovens adultos sobreviventes de câncer infantil há uma alta prevalência de sintomas de estresse pós-traumático (PTSS), como ficar reexperienciando a situação, hipervigilância e fuga de emoções. Estes sintomas podem persistir por anos após ter sido completado o tratamento (Wiener et al., 2006; Schwartz \& Drotar, 2006). Na relação entre PTSS e o comportamento de promoção de saúde, a incerteza é um mediador (Santacroce \& Lee, 2006).

A incerteza também é um fator de estresse, mas com o tempo pode ser experienciada por alguns sobreviventes como um profundo catalisador de mudanças positivas na identidade e na visão de mundo para novas formas de fé e crescimento espiritual (Parry, 2003).

Os aspectos psicossociais, como sair da casa dos pais, cobertura do seguro-saúde, casamento e emprego foram explorados em vários estudos, de formas diferentes, com metodologias diversas e com uso de grupo-controle ou não.

O fato de ter tido câncer na infância e ter sido tratado com cirurgia e/ou quimioterapia não interfere significativamente no processo geral de o sobrevivente deixar a casa dos pais e ter uma independência social na vida adulta, quando comparado ao grupo-controle. Sobreviventes de tumor de sistema nervoso central (TSNC) apresentaram mais dificuldade em deixar a casa dos pais (Koch et al., 2006).

Comparados com os irmãos, os adultos sobreviventes de câncer infantil têm significativamente menor taxa de cobertura de segurosaúde e mais dificuldade em obter o seguro (Park et al., 2005). Nos sobreviventes que não receberam radiação radioterápica, as taxas de cobertura de seguro-saúde, casamento e emprego foram similares em idade e sexo às médias nacionais (Pui et al., 2003; Langeveld et al., 2003; Stam et al., 2005; Nagarajan et al., 2003).

Comparados a um grupo-controle, os sobreviventes holandeses apresentaram baixa prevalência de casamento ou de vivência conjugal, resultado diferente do encontrado nos EUA (Langeveld et al., 2003; Stam et al., 2005).

No que concerne ao nível educacional, Langeveld et al. (2003) concluíram que os sobreviventes, apesar de apresentarem bom funcionamento e lidarem bem com a vida, apresentam prejuízos no desenvolvimento educacional, especialmente aqueles que receberam radiação craniana. Resultado diferente foi encontrado por Stam et al. (2005), em que os sobreviventes não diferiram do grupo-controle quanto ao nível educacional. Em uma amostra de 124 sobreviventes alemães foi encontrado que $65 \%$ têm desenvolvimento intelectual e mental adequado e 36\% têm sérios problemas de aprendizagem (Humpl, Fritsche, Bartels \& Gutjahr, 2001).

Os sobreviventes de leucemia, doença de Hodgkin e linfoma não-Hodgkin reportaram mais níveis de sofrimento somático e sintomas depressivos quando comparados ao grupo de irmãos, porém a maioria dos sobreviventes de câncer infantil é psicologicamente saudável. Em sobreviventes com déficits físicos funcionais, algumas perturbações sutis podem ser percebidas no ajustamento social (Zebrack et al., 2004; Zebrack et al., 2002; Joubert, Sadéghi, Devin, Laperrière \& Rodin, 2001).

Os jovens adultos sobreviventes de LLA e tumor de Wilms apresentam taxas de desordens psiquiátricas iguais à do grupo-controle (Machkie, Hill, Kondryn \& McNally, 2000). Os sobreviventes dinamarqueses, especialmente os de tumor cerebral, apresentaram mais risco de admissão em hospital psiquiátrico quando comparados à população em geral.

Em um estudo desenvolvido por Forsbach e Thompson (2003), foi possível perceber que há uma relação entre câncer infantil e relacionamento interpessoal adulto, e que quanto mais velho no diagnóstico, maior o impacto negativo nos relacionamentos.

O valor médio de QI dos sobreviventes foi considerado normal e sua deterioração se relaciona com a radiação craniana, a idade no momento do diagnóstico e a ocorrência de recidivas (Monleón et 
al., 2000). Nos sobreviventes de meduloblastoma foram identificados prejuízos em todos os domínios psicossociais, sobretudo nos domínios funcionais neuropsicológicos. Os sobreviventes e suas famílias não relataram prejuízo na qualidade de vida (Maddrey et al., 2005).

Os sobreviventes apresentaram escores globais de autoconceito significativamente mais baixos que os do grupo controle de irmãos (Seitzman et al., 2004)

\section{Saúde física/comprometimento físico}

Hudson et al. (2003) avaliaram o status de saúde nos sobreviventes a longo prazo. A saúde geral foi percebida pelos adultos sobreviventes como muito boa e $43,6 \%$ dos sobreviventes relataram ter tido prejuízo em um ou mais domínios avaliados no estudo.

Oeffinger et al. (2007) concluíram que os sobreviventes de câncer infantil sofrem oito vezes mais riscos de ter uma doença crônica ou alguma ameaça à vida do que o grupo-controle. Os sobreviventes de tumores ósseos, tumores de SNC e doença de Hodgkin são os que têm maiores riscos.

A fadiga experienciada por adultos sobreviventes de câncer infantil é igual à das outras pessoas da mesma idade (Langeveld, Grootenhuis, Voûte \& Van den Boss, 2003).

\section{Segunda neoplasia}

Seis estudos analisaram a incidência e o risco de segunda neoplasia nos adultos sobreviventes de câncer infantil.

O estudo desenvolvido na Holanda encontrou que os sobreviventes apresentaram, em geral, onze vezes mais risco que a população em geral (Cardous-Ubbink et al., 2007). No Reino Unido, um estudo mostrou que os sobreviventes apresentam quatro vezes mais riscos de desenvolver um segundo câncer que a população em geral (Hammal, Bell, Craft \& Parker, 2005). Apesar desta divergência, é unânime nas pesquisas a afirmação de que ter tido câncer na infância constitui risco para o desenvolvimento de uma segunda neoplasia.

$\mathrm{O}$ risco de segundo câncer foi maior entre os sobreviventes de tumores sólidos, câncer de tecidos moles, tumores de SNC e meningioma (CardousUbbink et al., 2007). Outros fatores também foram identificados - como ser do sexo feminino com doença de Hodgkin, ter idade entre 10 e 16 anos no primeiro diagnóstico sendo tratado com quimioterapia e radioterapia (Jazbec, Todorovski \& Jereb, 2007).

Pacientes tratados com quimioterapia sofrem duas vezes mais riscos de desenvolver um segundo câncer
(Cardous-Ubbink et al., 2007; Henderson et al., 2007; Pui et al., 2003).

O risco de câncer com o avanço da idade é maior, especialmente naqueles diagnosticados na infância (Hammal, Bell, Craft \& Parker, 2005; CardousUbbink et al., 2007).

\section{Fertilidade e reprodução}

A fertilidade dos adultos sobreviventes de câncer infantil foi estudada especificamente por dois artigos, porém esta temática é recorrente em vários outros artigos. Os sobreviventes demonstraram preocupação com a sua capacidade reprodutiva e em transferir geneticamente os problemas de saúde para os seus filhos (Zebrack, Casillas, Nohrm, Adams \& Zeltzer, 2004; Byrne et al., 2004; Forsbach \& Thompson, 2003).

Homens tratados com radioterapia cranial sem radioterapia espinhal antes dos 9 anos de idade têm menos chances de se tornar pais do que o grupocontrole (Byrne et al., 2004).

Filhos de sobreviventes têm aproximadamente duas vezes mais riscos de nascer pré-termo que os do grupo-controle. Além disso, os filhos de mulheres que receberam radiação pélvica têm risco de nascer com baixo peso (Signorello et al., 2006, Green et al., 2002).

\section{DISCUSSÃO}

A maioria dos artigos apresenta a proposta de conhecer o funcionamento psicossocial do sobrevivente de câncer infantil. Para isto, a maioria utiliza-se de métodos quantitativos, assim como comparação com grupo-controle, com a população em geral e com os irmãos.

Este quadro atual é esperado, uma vez que os sobreviventes de câncer infantil estão crescendo e se tornando adultos e as possíveis consequências a longo prazo ainda são pouco conhecidas.

Por outro lado, nota-se uma escassez de pesquisas de cunho qualitativo, que investiguem a vivência do adulto sobrevivente de câncer infantil, ou seja, a forma como esse adulto ressignifica o fato de ter tido câncer na infância.

Observa-se uma grande heterogeneidade dos estudos, porém, apesar dessa diversidade, percebe-se certa tendência a apontar que os sobreviventes apresentam funcionamento psicossocial sem nenhum tipo de comprometimento, com algumas exceções referentes ao diagnóstico inicial (TSNC) e ao uso da radioterapia. 
No entanto, apesar dos resultados positivos encontrados, as pesquisas ainda estão mais focadas na perspectiva teórica centrada na deficiência e no mau ajustamento do que no enfrentamento. Inclusive, em sua maioria, partem da hipótese que os sobreviventes têm funcionamento psicossocial comprometido em relação ao grupo comparado. Este enfoque prediz que a experiência de uma doença crônica está pretensamente associada com mau ajustamento (Eiser, 1998; Eiser, Hill \& Vance, 2000). Este fato é bastante visível na discussão dos trabalhos em que não são encontradas diferenças significativas entre o grupo de sobreviventes e o grupo-controle, e justificam-se esses resultados como sendo problemas metodológicos ou decorrentes de uma amostra pequena.

Os estudos também apontaram que a forma como os sobreviventes se veem e se percebem exerce maior influência na sua qualidade de vida do que o status médico de saúde. Este fato pode ser um foco importante nas futuras propostas de intervenção na promoção de saúde dos sobreviventes.

Ser sobrevivente de câncer infantil implica em maior risco de desenvolver uma segunda neoplasia e parece influenciar a fertilidade na vida adulta. Esta temática foi recorrente nos artigos, o que mostra a preocupação de compreender e conhecer as variáveis de risco e assim poder amenizar o impacto dessas variáveis em tratamentos posteriores.

Foi possível perceber, nos estudos, uma grande diversidade no que concerne à amostra estudada. A idade no momento do diagnóstico foi delimitada até os 21 anos, acarretando, assim, uma amostra composta por adolescentes e por adultos. Entendemos que ser adulto sobrevivente de câncer infantil e ser adulto sobrevivente de câncer na adolescência envolvem aspectos diferentes, uma vez que cada fase do desenvolvimento é uma fase da vida peculiar, com várias características e nuanças próprias.

Observou-se também uma grande diversidade com relação ao tempo de sobrevivência e a quando esse tempo começa a ser contado, se no diagnóstico ou no fim do tratamento. Este fato acarreta dificuldades em se delimitar quando alguém pode ser considerado um sobrevivente. Esta temática vem sendo amplamente discutida, mas ainda é uma questão que gera controvérsia.

Nesse sentido, é necessário que os pesquisadores, em seus trabalhos, definam o que está sendo chamado de sobrevivente e que deixem bastante explícitas questões como a idade no diagnóstico, no momento da pesquisa e o tempo livre da doença.

Meadows (2003) propõe que o sobrevivente de câncer pediátrico seja definido como alguém que tenha sobrevivido ao menos 5 anos desde a última evidência da doença e que tenha concluído o tratamento há pelo menos 2 anos.

Percebe-se que, de uma forma geral, os sobreviventes são comparados à média da população em geral, a um grupo controle ou a grupo de irmãos. Quando comparados à população em geral, implica em dizer que os resultados diferentes encontrados estão diretamente relacionados ao fato de ter tido câncer, excluindo, assim, os outros aspectos da vida do indivíduo. Com relação a comparar com irmãos, vários estudos apontam que os irmãos também são afetados pela experiência do câncer e que por isso não seriam um grupo controle adequado (Eiser, 1998; Cuttini et al., 2003).

Eiser, Hill e Vance (2000) sugerem que sejam realizados estudos longitudinais, em que se teria a possibilidade de conhecer as mudanças no ajustamento e no funcionamento psicossociais, ao longo do tempo, dos pacientes curados de câncer na infância.

No levantamento realizado por Langeveld, Stam, Grootenhuis e Last (2002) sobre qualidade de vida de adultos sobreviventes de cancer infantil, os artigos são provenientes da América do Norte e da Europa, havendo apenas 1 (um) trabalho fora deste âmbito, provindo da Austrália. Este dado corrobora esta revisão bibliográfica, em que a maioria dos artigos foram desenvolvidos nos Estados Unidos, não sendo encontrado nenhum proveniente da América Latina. Desta forma, perecebe-se uma carência e a necessidade de se investigar o adulto sobrevivente de câncer infantil em outros países, em diferentes contextos culturais.

Ao finalizar o tratamento, os sobreviventes iniciam o acompanhamento no serviço específico. Como essa prática é bastante recente, os pesquisadores estão investigando a melhor forma de ser estruturado esse serviço, cujo papel e funções são discutidos nos artigos teóricos.

\section{CONSIDERAÇÕES FINAIS}

Com o levantamento, foi possível perceber que o sobrevivente adulto de câncer infantil está sendo cada vez mais alvo de pesquisa e que há um interesse da comunidade científica em avaliar e constatar as consequências deste tratamento a longo prazo. Não obstante, nas bases de dados pesquisadas e segundo os critérios de inclusão utilizados não foram encontrados trabalhos nacionais, o que suscita a necessidade de se investigar esta temática. 
Além disso, o trabalho levanta a necessidade de conciliar a pesquisa e a prática assistencial, uma vez que para conhecer e acompanhar os adultos sobreviventes de câncer infantil faz-se necessário discutir e aprimorar a ideia do serviço para acompanhamento.

Meadows (2003) propõe que o programa de acompanhamento para sobreviventes de câncer infantil deve: prover cuidado clínico e proporcionar um ambiente para conduzir pesquisa de alta qualidade, pois assim poderá dar suporte, aconselhamento e orientação antecipada; ser dirigido multidisciplinarmente, propiciando assim uma educação para saúde e podendo detectar precocemente efeitos a longo prazo conforme a terapia utilizada; e padronizar o serviço de acompanhamento para avaliar os efeitos das modificações ocorridas durante $o$ tratamento.

\section{REFERÊNCIAS}

Blaauwbroek, R., Stant, A. D., Groenier, K. H., Kamps, W.A., Meyboom, B., \& Postma, A. (2007). Health-related quality of life and adverse late effects in adult (very) long-term childhood cancer survivors. European Journal of Cancer, 43, 122-130.

Byrne, J., Fears, T. R., Mills, J. L., Zeltzer, L. K., Sklar, C., Meadows, A. T., Reaman, G. H., \& Robison, L. L. (2004). Fertility of longterm male survivors of acute lymphoblastic leukemia diagnosed during childhood. Pediatric Blood \& Cancer, 42, 364-372.

Cardous-Ubbink, M.C., Heinen, R. C., Bakker, P. J. M., Van Den Berg, H., Oldenburger, F., Caron, H.N., Voûte, P.A., \& Van Leeuwen, F.E. (2007). Risk of second malignancies in long-term survivors of childhood cancer. European Journal of Cancer, 43, 351-362.

Cella, D.F., Tan, C., Sullivan, M., \& Weinstock, L. (1987). Identifying survivors of pediatric Hodgkin's disease who need psychological interventions. Journal of Psychosocial Oncology, 5(4), 83-96.

Cuttini, M., Da Frè, M., Haupt, R., Déb, G., Baronci, C., Tamaro, P., Zebrack, B., Zeltzer, L. K., Whitton, J., Berkopw, R., \& Chesler, M.A. (2003) Survivors of childhood câncer: using siblings as a control group. Pediatrics, 112, 1454-1455.

Eiser, C. (1998) Practitioner Review: Long-term consequences of childhood cancer. Journal of Child Psychology and Psychiatry, and Allied Disciplines, 39(5), 621-633.

Eiser, C., Hill, J. J. \& Vance, Y. H. (2000). Examining the Psychological consequences of surviving childhood cancer: systematic review as a research method in pediatric psychology. Journal of Pediatric Psychology, 25(6), 449-460.

Forsbach, T. \& Thompson, A. (2003). The impact of childhood cancer on adult survivors' interpersonal relationship. Child Care in Practice, 9(2), 117-128.

Garre, M.L., Gandus, S., Cesana, B., Haupt, R., De Bernardi, B., Comelli, A., Ferrando, A., Stella, G., Vitali, M.L. \& Picco, P. (1994). Health status of long-term survivors after cancer in childhood. Results of an uniinstitutional study in Italy. The
American journal of pediatric hematology/oncology, 16(2), 143152.

Green, D.M., Whitton, J.A., Stovall, M., Mertens, A.C., Donaldson, S.S., Ruymann, F.B., Pendergrass, T. W. \& Robison, L.L. (2002). Pregnancy outcome of female survivors of childhood cancer: A report from the Childhood Cancer Survivor Study. American Journal of Obstetrics and Gynecology, 187, 1070-80.

Hammal, D.M.; Bell, C.L.; Craft, A.W.; Parker, L. (2005). Second Primary Tumors in children and Young Adults in the North of England (1968-99). Pediatric Blood \& Cancer, 45, 155-161.

Henderson, T.O., Whitton, J., Stovall, M., Mertens, A.C., Mitby, P., Friedman, D., Strong, L.C., Hammond, S., Neglia, J. P., Meadows, A.T., Robison, L. \& Diller, L. (2007). A secondary sarcomas in childhood cancer survivors: a report from the childhood cancer survivor study. Journal of the National Cancer Institute 2 99, 300-8.

Hewitt, M., Weiner, S., Simone, J. (2003). Childhood cancer survivorship improving care and quality of life. National Academy Press.

Hudson, M.M.; Mertens, A.C.; Yasui, Y.; Hobbie, W. ; Chen, H., et al (2003). Health status of adult long-term survivors of childhood cancer: a report from the childhood cancer survivor study. JAMA: The Journal of the American Medical Association, 290(12), 15831592.

Humpl, T., Fritsche, M., Bartels, U., \& Gutjahr, P. (2001). Survivors of Childhood Cancer for more than twenty years. Acta Oncológica, 40(1), 44-49.

Instituto Nacional do Câncer (2007). Particularidades do Câncer Infantil. Recuperado em 26 de setembro de 2007 em http://www.inca.gov.br/conteudo_view.asp?id=343

Jazbec, J., Todorovski, L., Jereb, B. (2007). Classification tree analysis of second neoplasms in survivors of childhood cancer. $B M C$ Cancer, 2, 7-27.

Joubert, D., Sadéghi, M. R., Devins, G. M., Laperrière, N., \& Rodin, G. (2001). Physical sequelae and self-perceived attachment in adult survivors of childhood cancer. Psycho-oncology, 10, 284292.

Koch, S. V., Kejs, A. M. T., Engholm, G., Moller, H., Johansen, C., \& Schmiegelow, K. (2006). Leaving home after cancer in childhood: a measure of social independence in early adulthood. Pediatric Blood \& Cancer, 47, 61-70.

Langeveld, N.E., Stam, H., Grootenhuis, M.A. \& Last, B. F. (2002). Quality of life in young adult survivors of childhood cancer. Support Care Cancer, 10, 579-600.

Langeveld, N.E., Grootenhuis, M.A., Voûte, P.A., De Haan, R. J., \& Van Den Bos, C. (2004). Quality of life, self-esteem and worries in young adult survivors of childhood cancer. Psycho-oncology, $13,867-881$

Langeveld, N.E., Ubbink, M.C., Last, B. F., Grootenhuis, M.A., Voûte, P.A., \& De Haan, R. J. (2003). Educational achievement, employment and living situation in long-term young adult survivors of childhood cancer in the Netherlands. Psychooncology, 12, 213-225.

Langeveld, N.E., Grootenhuis, M.A., Voûte, P.A., de Haan, R.J., \& Van Den Bos, C. (2003). No excess fatigue in young adult survivors of childhood cancer. European Journal of Cancer, 39, 204-214. 
Machkie, E., Hill, J., Kondryn, H., \& McNally, R. (2000). Adult psychosocial outcomes in long-term survivors of acute lymphoblastic leukaemia and Wilms' tumour: a controlled study. The Lancet, 355, 1310-14.

Maddrey, A. M., Bergeron, J. A., Lombardo, E. R., McDonald, N:K., Mulne, A. F., Barenberg, P. D., \& Bowers, D. C. (2005). Neuropsychological performance and quality of life of 10 year survivors of childhood medulloblastoma. Journal of NeuroOncology, 72, 245-253.

Meadows, A. T. (2003) Pediatric cancer survivors: past history and future challenges. Current Problems in Cancer, 27(3), 112-126.

Mellette, S.J., \& Franco, P. C. (1987). Psychosocial barriers to employment of the cancer survivor. Journal of Psychosocial Oncology, 5(4), 97-115.

Monleón, M. C. B., Andreu, J. A. L., Estellés, I. S., Castaño, M.H., Aguilar, J. G., Delgado, F. M., \& Tortajada, J. F. (2000). Secuelas psicológicas en los supervivientes a largo plazo de câncer. Anales Españoles de Pediatría, 53(6), 553-560.

Mulhern, R.K., Tyc, V. L., Phipps, S., Crom, D., Barclay, D., Greenwald, C., Hudson, M., \& Thompson, E.L. (1995). Healthrelated behaviors of survivors of childhood cancer. Medical and pediatric oncology, 25(3), 159-65.

Nagarajan, R., Neglia, J. P., Clohisy, D. R., Yasui, Y., Greenberg, M., Hudson, M., Zevon, M.A., Tersak, J.M., Ablin, A., \& Robison, L.L. (2003). Education, Employment, Insurance, and Marital Status among 694 survivors of Pediatric Lower Extremity Bone Tumors: a report from the childhood cancer survivor study. Cancer, 97, 2554-64.

Oeffinger, K. C.,Mertens, A. C., Sklar, C. A., Kawashima, T., Hudson, M. M., Meadows, A. T., Friedman, D. L.,Marina, N., Hobbie, W., Kadan-Lottick, N.S., Schwartz, C. L., Leisenring, W., \& Robison, L.L. (2007). Chronic Health conditions in Adult Survivors of childhood Cancer. The New England Journal of Medicine, 355, 1572-82.

Park, E. R., Li, F. P., Liu, Y., Emmons, K. M., Ablin, A., Robison, L. L., \& Mertens, A. C. (2005). Health Insurance coverage in survivors of Childhood Cancer: The Childhood Cancer Survivor Study. Journal of Clinical Oncology, 23(36), 9187-9197.

Parry, C. (2003). Embracing uncertainty: an exploration of the experiences of childhood cancer survivors. Qualitative Health Research, 13(1), 227-246.

Pemberger, S., Jagsch, R., Frey, E., Felder- Puig, R., Gadner, H., Kryspin-Exner, I., \& Topf, R. (2005). Quality of Life in long-term Childhood Cancer Survivors and the Relation of Late Effects and Subjective Well-Being. Support Care Center, 13, 49-56.

Pui, C-H, Cheng, C., Leung, W., Rai, S.N., Rivera, G.K., Sandlund, J.T., Ribeiro, R.C., Relling, M.V., Kun, L.E., Evans, W.E., \& Hudson, M.M. (2003). Extended Follow-up of Long-Term Survivors of Childhood Acute Lymphoblastic Leukemia. The New England Journal of Medicine, 349, 640-649.

Rodrigues, K. E., \& Camargo, B. (2003). Diagnóstico precoce do câncer infantil: responsabilidade de todos. Revista da Associação Médica Brasileira, 49(1), 29-34.

Ross, L., Johansen, C., Dalton, S.O., Mellemkjaer, L., Thomasse, L.H., Mortensen, P. B., \& Oslen, J.H. (2003). Psychiatric Hospitalizations among survivors of cancer in childhood or adolescence. The New England Journal of Medicine, 349, 650657.
Santacroce, S. J., \& Lee, Y.(2006).Uncertainty, Posttraumatic Stress, and Health Behavior in Young Adult Childhood Cancer Survivors. Nursing Research, 55(4), 259-266.

Schwartz, C. L. (2003). Health Status of Childhood Cancer survivors: cure is more than the eradication of cancer. JAMA: The Journal of the American Medical Association, 290(12), 1641-1643.

Schwartz, L., \& Drotar, D., (2006). Posttraumatic stress and related impairment in survivors of childhood cancer in early adulthood compared to healthy peers. Journal of Pediatric Psychology, 31(4), 356-366.

Seitzman, R. L., Glover, D.A., Meadows, A. T., Mills, J. L., Nicholson, H. S., Robison, L. L., Byrne, J., \& Zeltzer, L. K. (2004). Self-concept in adult survivors of childhood acute lymphoblastic leukemia: A Cooperative Children's Cancer Group and National Institutes of Health Study. Pediatric Blood \& Cancer, 42: 230-240.

Signorello, L. B., Cohen, S. S., Bosetti, C., Stovall, M., Kasper, C. E., Weathers, R. E., Whitton, J. A., Green, D. M., Donaldson, S. S., Mertens, S. C., Robison, L. L., \& Boice Jr., J. D. (2006). Female survivors of childhood cancer: preterm birth and low birth weight among their children. Journal of the National Cancer Institute, 98(20), 1453-1461.

Stam, H; Grootenhuis, M. A., \& Last, B. F. (2005). The course of life of survivors of childhood cancer. Psycho-oncology, 14: 227-238.

Stam, H.; Grootenhuis, M. A.; Caron, H. N., \& Last, B.F. (2006). Quality of life and current coping in young adult survivors of childhood cancer: positive expectations about the further course of the disease were correlated with better quality of life. Psychooncology, 15, 31-43.

Taylor, A., Hawkins, M., Griffiths, A., Davies, H., Douglas, C., Jenney, M., Wallace, W. H. B., \& Levitt, G. (2004). Long-term follow-up of survivors of Childhood cancer in the UK. Pediatric Blood \& Cancer, 42, 161-168.

Teeter, M.A., Holmes, G.E., Holmes, F.F., \& Baker, A.B. (1987). Decisions about marriage and family among survivors of childhood cancer. Journal of Psychosocial Oncology, 5(4), 59-68.

Voûte, P.A. (1999). Câncer na infância. Tradução Arion Caio Paulino. In: União Internacional contra o câncer. Manual de Oncologia Clínica. Tradução da $6^{\mathrm{a}}$ edição original. São Paulo, 531-534.

Weigers, M.E., Chesler, M.A., Zebrack, B.J., \& Goldman, S. (1998). Self-reported worries among long-term survivors of childhood cancer and their peers. Journal of Psychosocial Oncology, 16(2), $1-23$.

Wiener, L., Battles, H., Bernstein,D., Long, L., Derdak, J., Mackall, C.L., \& Mansky, P.J. (2006). Persistent psychological distress in long-term survivors of pediatric sarcoma: the experience at a single institution. Psycho-oncology, 15, 898-910.

Zebrack, B.J., \& Chesler, M. A. (2002). Quality of life in childhood cancer survivors. Psycho-oncology, 11, 132-141.

Zebrack, B.J., Casillas, J., Nohr, L., Adams, H., \& Zeltzer, L.K. (2004). Fertility issues for young adult survivors of childhood cancer. Psycho-oncology, 13, 689-699.

Zebrack, B. J., Gurney, J. G., Oeffinger, K., Whitton, J., Packer, R. J., Mertens, A., Turk, N., Castleberry, R., Dreyer, Z., Robinson, L. L., \& Zeltzer, L. (2004). Psychological outcomes in ling-term survivors of childhood brain cancer: a report from the childhood cancer survivor study. Journal of Clinical Oncology, 22(6), 9991006. 
Zebrack, B.J., Zeltzer, L.K., Whitton, J., Mertens, A.C., Odom, L., Berkow, R., \& Robison, L.L. (2002). Psychological outcomes in long-term survivors of childhood leukemia, Hodgkin's disease, and Non-Hodgkin's Lymphoma: a report from the childhood Cancer Survivor study. Pediatrics, 110, 42-52.

Endereço para correspondência :

Shirley Santos Teles, Rua Pedro Lisboa, n.122, Residencial Parque dos Coqueiros, Bairro: Inácio Barbosa, CEP 49040-800, Aracaju - SE, Brasil. E-mail: shirleyteles@ gmail.com 